\title{
Semi-classical solutions of perturbed elliptic system with general superlinear nonlinearity
}

Fangfang Liao ${ }^{1,2}$, Xianhua Tang ${ }^{1 *}$, Jian Zhang ${ }^{1}$ and Dongdong Qin ${ }^{1}$

\section{"Correspondence:}

tangxh@mail.csu.edu.cn

'School of Mathematics and

Statistics, Central South University,

Changsha, Hunan 410083, P.R. China

Full list of author information is

available at the end of the article

\begin{abstract}
This paper is concerned with the following perturbed elliptic system: $-\varepsilon^{2} \Delta u+V(x) u=W_{v}(x, u, v), x \in \mathbb{R}^{N},-\varepsilon^{2} \Delta v+V(x) v=W_{u}(x, u, v), x \in \mathbb{R}^{N}, u, v \in H^{1}\left(\mathbb{R}^{N}\right)$, where $V \in C\left(\mathbb{R}^{N}, \mathbb{R}\right)$ and $W \in C^{1}\left(\mathbb{R}^{N} \times \mathbb{R}^{2}, \mathbb{R}\right)$. Under some mild conditions on the potential $V$ and nonlinearity $W$, we establish the existence of nontrivial semi-classical solutions via variational methods, provided that $0<\varepsilon \leq \varepsilon_{0}$, where the bound $\varepsilon_{0}$ is formulated in terms of $N, V$, and $W$.
\end{abstract}

MSC: $35 J 10 ; 35 J 20$

Keywords: semi-classical solutions; perturbed elliptic system; generalized linking theorems

\section{Introduction}

The goal of this paper is to establish the existence of semi-classical solutions to the following perturbed elliptic system of Hamiltonian form:

$$
\begin{cases}-\varepsilon^{2} \Delta u+V(x) u=W_{v}(x, u, v), & x \in \mathbb{R}^{N}, \\ -\varepsilon^{2} \Delta v+V(x) v=W_{u}(x, u, v), & x \in \mathbb{R}^{N}, \\ u, v \in H^{1}\left(\mathbb{R}^{N}\right), & \end{cases}
$$

where $u, v: \mathbb{R}^{N} \rightarrow \mathbb{R}, \varepsilon>0$ is a small parameter, and $V: \mathbb{R}^{N} \rightarrow \mathbb{R}$ and $W: \mathbb{R}^{N} \times \mathbb{R}^{2} \rightarrow \mathbb{R}$ satisfy the following basic assumptions, respectively:

(V0) $V \in C\left(\mathbb{R}^{N}\right)$ and there exists a $b>0$ such that the set $\mathcal{V}_{b}:=\left\{x \in \mathbb{R}^{N}: V(x)<b\right\}$ has finite measure;

(V1) $V(x) \geq \min V=0$;

(W1) $W \in C^{1}\left(\mathbb{R}^{N} \times \mathbb{R}^{2}\right)$, and there exist constants $p_{0} \in\left(2,2^{*}\right)$ and $C_{0}>0$ such that

$$
\left|W_{z}(x, z)\right| \leq C_{0}\left(1+|z|^{p_{0}-1}\right), \quad \forall(x, z) \in \mathbb{R}^{N} \times \mathbb{R}^{2}, z=(u, v)
$$

here and in the sequel $2^{*}:=2 N /(N-2)$ if $N \geq 3$ and $2^{*}:=+\infty$ if $N=1$ or 2 ;

(W2) $\left|W_{z}(x, z)\right|=o(|z|)$, as $|z| \rightarrow 0$, uniformly in $x \in \mathbb{R}^{N}$.

For the case $\varepsilon=1$, the interest in the study of various qualitative properties of the solutions has steadily increased in recent years. In a bounded smooth domain $\Omega \subset \mathbb{R}^{N}$, similar systems have been extensively studied; see, for instance, [1-6] and the references therein. The problem set on the whole space $\mathbb{R}^{N}$ was considered recently in some works. The first

\section{6ringer}

(c) 2014 Liao et al.; licensee Springer. This is an Open Access article distributed under the terms of the Creative Commons Attribution License (http://creativecommons.org/licenses/by/4.0), which permits unrestricted use, distribution, and reproduction in any medium, provided the original work is properly credited. 
difficulty of such a type of problem is the lack of the compactness of the Sobolev embedding. A usual way to recover this difficulty is choosing suitable working space which has compact embedding property, for example, the radially symmetric functions space; see [7-9]. The second difficulty is that the energy functional is strongly indefinite different from the single equation case, and so the dual variational methods are involved to avoid this difficulty; see $[10,11]$. Recently, with the aid of the linking arguments in [12-14], the existence of solutions or multiple solutions were obtained with periodic potential and nonlinearity, see [15-19] and the references therein.

For the problem with a small parameter $\varepsilon>0$, it is called the semi-classical problem, which describes the transition between of quantum mechanics and classical mechanics with the parameter $\varepsilon$ goes to zero. There is much literature dealing with the existence of semi-classical solutions to the single particle equation

$$
-\varepsilon^{2} \Delta u+V(x) u=g(x, u), \quad x \in \mathbb{R}^{N}, u \in H^{1}\left(\mathbb{R}^{N}\right),
$$

under various hypotheses on the potential $V$ and the nonlinearity $g$; for example, see [2027] and references therein. In a very recent paper [28], Lin and Tang developed a direct and simple approach to show the existence of semi-classical solutions for the single particle equation (1.2) with $V$ satisfying (V0) and (V1). It is well known that the extension of these results of single equation to a system of equations presents some difficulties. One of the main difficulties is that the energy functional associated with (1.1) is strongly indefinite, so the approach used in the single equation is not applicable to system (1.1).

Inspired by the single equation, there are a few works considering the perturbed elliptic systems; see [11, 29-35] and references therein. To the best of our knowledge, the first approach to the singular perturbed system in a bounded domain, with Neumann boundary, and $V(x) \equiv 1$ appeared in [11] by means of a dual variational formulation of the problem. Moreover, in [33], Sirakov and Soares considered the superquadratic case by using dual variational methods. In [31], Ramos and Tavares considered the following problem:

$$
\left\{\begin{array}{l}
-\varepsilon^{2} \Delta u+V(x) u=g(v), \quad x \in \Omega, \\
-\varepsilon^{2} \Delta v+V(x) v=f(u), \quad x \in \Omega, \\
u(x)=0 \quad \text { and } \quad v(x)=0 \quad \text { on } \partial \Omega
\end{array}\right.
$$

where $\Omega$ is a domain of $\mathbb{R}^{N}, f$ and $g$ are power functions, superlinear but subcritical at infinity. The authors established the existence of positive solutions which concentrate, as $\varepsilon \rightarrow 0$, on a prescribed finite number of local minimum points of the potential $V$; also see [30].

Since Kryszewski and Szulkin [6] proposed the generalized linking theorem for the strongly indefinite functionals in 1998, Li and Szulkin [13], Bartsch and Ding [12] gave several weaker versions, which provide another effective way to deal with such problems. With the aid of the generalized linking theorem, Xiao et al. [34] studied the asymptotically quadratic case and obtained the existence of multiple solutions. For the superquadratic case with magnetic potential, we refer the reader to [35] and references therein.

In the aforementioned references, it always was assumed that $W$ satisfies a condition of the type of Ambrosetti-Rabinowitz, that is, 
(AR) there is a $\mu>2$ such that

$$
0<\mu W(x, z) \leq W_{z}(x, z) \cdot z, \quad \forall(x, z) \in \mathbb{R}^{N} \times \mathbb{R}^{2}, z \neq 0 ;
$$

together with a technical assumption that there is $v>2 N /(N+2)$ such that

$$
\left|W_{z}(x, z)\right|^{v} \leq c\left[1+W_{z}(x, z) \cdot z\right], \quad \forall(x, z) \in \mathbb{R}^{N} \times \mathbb{R}^{2}
$$

or the superquadratic condition

$$
\lim _{|z| \rightarrow \infty} \frac{|W(x, z)|}{|z|^{2}}=\infty, \quad \text { uniformly in } x \in \mathbb{R}^{N}
$$

and a condition of the type of Ding-Lee [36];

(DL) $\widetilde{W}(x, z):=\frac{1}{2} W_{z}(x, z) z-W(x, z)>0$ for $z \neq 0$ and there exist $c_{0}>0$ and $\kappa>\max \{1, N / 2\}$ such that

$$
\left|W_{z}(x, z)\right|^{\kappa} \leq c_{0}|z|^{\kappa} \widetilde{W}(x, z) \quad \text { for large }|z| .
$$

As is well known, conditions (1.3) and (1.4) have been successfully applied to Hamiltonian systems, to periodic Schrödinger systems, and to diffusion systems; see [37, 38] and so on. We refer the reader to [39-43] and the references therein where the condition (AR) was weakened by more general superlinear conditions. Condition (DL) was firstly given for a single Schrödinger equation by Ding and Lee [36]. Soon after, this condition was generalized by Zhang et al. [44].

Observe that conditions (1.5) and $W(x, z)>0, \forall z \neq 0$ in (AR) or $\widetilde{W}(x, z)>0, \forall z \neq 0$, in (DL) play an important role in showing that any Palais-Smale sequence or Cerami sequence is bounded in the aforementioned works. However, there are many functions which do not satisfy these conditions, for example,

$$
W(x, u, v)=|u+v|^{\varrho}, \quad \varrho \in\left(2,2^{*}\right)
$$

or

$$
W(x, u, v)=(u+v)^{2} \sqrt{u^{2}+v^{2}} .
$$

Motivated by these works, in the present paper, we shall establish the existence of semiclassical solutions of system (1.1) with a weaker superlinear condition via the generalized linking theorem. To state our results, in addition to the basic hypotheses, we make the following assumptions:

(W3) there exist $a_{0}>0$ and $p \in\left(2,2^{*}\right)$ such that

$$
W(x, u, v) \geq a_{0}|u+v|^{p}, \quad \forall(x, u, v) \in \mathbb{R}^{N} \times \mathbb{R}^{2} ;
$$

(W4) $\widetilde{W}(x, z) \geq 0, \forall(x, z) \in \mathbb{R}^{N} \times \mathbb{R}^{2}$, and there exist $R_{0}>0, a_{1}>0$, and $\kappa>\max \{1, N / 2\}$ such that

$$
\left|W_{u}(x, z)+W_{v}(x, z)\right| \leq \frac{b}{3}|z|, \quad \forall(x, z) \in \mathbb{R}^{N} \times \mathbb{R}^{2},|z| \leq R_{0}
$$


and

$$
\left|W_{u}(x, z)+W_{v}(x, z)\right|^{\kappa} \leq a_{1}|z|^{\kappa} \tilde{W}(x, z), \quad \forall(x, z) \in \mathbb{R}^{N} \times \mathbb{R}^{2},|z| \geq R_{0} .
$$

In the present paper, we make use of the techniques developed in $[28,45]$ to obtain the existence of semi-classical solutions for system (1.1) when $\varepsilon \leq \varepsilon_{0}$, where the bound $\varepsilon_{0}$ is formulated in terms of $N, V$, and $W$.

Since $(p-2) N-2 p<0$, we can choose a $h_{0} \geq 1$ such that

$$
\frac{(p-2) \omega_{N}}{4 N a_{0}^{2 /(p-2)}}\left[\frac{N^{3}+2(N+2)}{2(N+2) p\left(1-2^{-N}\right)^{2}}\right]^{p /(p-2)} h_{0}^{[(p-2) N-2 p] /(p-2)} \leq \frac{b^{(2 \kappa-N) / 2}}{3^{\kappa} \sqrt{2} a_{1}\left(\gamma_{2} * \gamma_{0}\right)^{N}} .
$$

Let $x_{0} \in \mathbb{R}^{N}$ be such that $V\left(x_{0}\right)=0$. Then we can choose $\lambda_{0}>1$ such that

$$
\sup _{\lambda^{1 / 2}|x| \leq 2 h_{0}}\left|V\left(x_{0}+x\right)\right| \leq h_{0}^{-2}, \quad \forall \lambda \geq \lambda_{0}
$$

Let $E$ be a Hilbert space as defined in Section 2 and $\lambda=\varepsilon^{-2}$. Under assumptions (V0), (V1), (W1), and (W2), the functional

$$
\Phi_{\lambda}(z)=\int_{\mathbb{R}^{N}}(\nabla u \cdot \nabla v+\lambda V(x) u v) \mathrm{d} x-\lambda \int_{\mathbb{R}^{N}} W(x, u, v) \mathrm{d} x, \quad \forall z=(u, v) \in E,
$$

is well defined. Moreover, $\Phi_{\lambda} \in C^{1}(E, \mathbb{R})$ and for all $z=(u, v), \zeta=(\varphi, \psi) \in E$

$$
\begin{aligned}
\left\langle\Phi_{\lambda}^{\prime}(z), \zeta\right\rangle= & \int_{\mathbb{R}^{N}}[\nabla u \cdot \nabla \psi+\nabla v \cdot \nabla \varphi+\lambda V(x)(u \psi+v \varphi)] \mathrm{d} x \\
& -\lambda \int_{\mathbb{R}^{N}}\left[W_{u}(x, u, v) \varphi+W_{v}(x, u, v) \psi\right] \mathrm{d} x .
\end{aligned}
$$

We are now in a position to state the main results of this paper.

Theorem 1.1 Assume that $V$ and $W$ satisfy (V0), (V1), (W1), (W2), (W3), and (W4). Then for $0<\varepsilon \leq \lambda_{0}^{-1 / 2},(1.1)$ has a solution $\left(u_{\varepsilon}, v_{\varepsilon}\right)$ such that $0<\Phi_{\varepsilon^{-1 / 2}}\left(u_{\varepsilon}, v_{\varepsilon}\right) \leq \frac{b^{(2 \kappa-N) / 2}}{3^{\kappa} \sqrt{2} a_{1}\left(\gamma_{2^{*}} \gamma_{0}\right)^{N}} \varepsilon^{N-2}$, and

$$
\int_{\mathbb{R}^{N}} \widetilde{W}\left(x, u_{\varepsilon}, v_{\varepsilon}\right) \mathrm{d} x \leq \frac{b^{(2 \kappa-N) / 2}}{3^{\kappa} \sqrt{2} a_{1}\left(\gamma_{2^{*}} \gamma_{0}\right)^{N}} \varepsilon^{N}
$$

Theorem 1.2 Assume that $V$ and $W$ satisfy(V0), (V1), (W1), (W2), (W3), and (W4). Then for $\lambda \geq \lambda_{0},(2.1)$ has a solution $\left(u_{\lambda}, v_{\lambda}\right)$ such that $0<\Phi_{\lambda}\left(u_{\lambda}, v_{\lambda}\right) \leq \frac{b^{(2 \kappa-N) / 2}}{3^{\kappa} \sqrt{2} a_{1}\left(\gamma_{2^{*}} \gamma_{0}\right)^{N}} \lambda^{1-N / 2}$, and

$$
\int_{\mathbb{R}^{N}} \widetilde{W}\left(x, u_{\lambda}, v_{\lambda}\right) \mathrm{d} x \leq \frac{b^{(2 \kappa-N) / 2}}{3^{\kappa} \sqrt{2} a_{1}\left(\gamma_{2^{*}} \gamma_{0}\right)^{N}} \lambda^{-N / 2}
$$

Before proceeding to the proofs of these theorems, we give two examples to illustrate the assumptions.

Example 1.3 $W(x, u, v)=h(x)|u+v|^{\varrho}$ satisfies (W1)-(W4), where $\varrho \in\left(2,2^{*}\right), h \in C\left(\mathbb{R}^{N}\right)$ with $\inf _{\mathbb{R}^{N}} h>0$. 
Example 1.4 $W(x, u, v)=h(x)(u+v)^{2} \sqrt{2 u^{2}+v^{2}}$ satisfies (W1)-(W4), where $h \in C\left(\mathbb{R}^{N}\right)$ with $\inf _{\mathbb{R}^{N}} h>0$.

The rest of the paper is organized as follows. In Section 2, we provide a variational setting. In Section 3, we give the proofs of our theorems.

\section{Variational setting}

Letting $\varepsilon^{-2}=\lambda$, (1.1) is rewritten as

$$
\begin{cases}-\Delta u+\lambda V(x) u=\lambda W_{v}(x, u, v), & x \in \mathbb{R}^{N}, \\ -\Delta v+\lambda V(x) v=\lambda W_{u}(x, u, v), & x \in \mathbb{R}^{N}, \\ u, v \in H^{1}\left(\mathbb{R}^{N}\right) . & \end{cases}
$$

Let

$$
\begin{aligned}
& E_{V}=\left\{u \in H^{1}\left(\mathbb{R}^{N}\right): \int_{\mathbb{R}^{N}} V(x) u^{2} \mathrm{~d} x<+\infty\right\}, \\
& (u, v)_{\lambda V}=\int_{\mathbb{R}^{N}}[\nabla u \cdot \nabla v+\lambda V(x) u v] \mathrm{d} x, \quad \forall u, v \in E_{V}
\end{aligned}
$$

and

$$
\|u\|_{\lambda V}=\left\{\int_{\mathbb{R}^{N}}\left[|\nabla u|^{2}+\lambda V(x) u^{2}\right] \mathrm{d} x\right\}^{1 / 2}, \quad \forall u \in E_{V} .
$$

Analogous to the proof of [46, Lemma 1], by using (V0), (V1), and the Sobolev inequality, one can demonstrate that there exists a constant $\gamma_{0}>0$ independent of $\lambda$ such that

$$
\|u\|_{H^{1}\left(\mathbb{R}^{N}\right)} \leq \gamma_{0}\|u\|_{\lambda V}, \quad \forall u \in E_{V}, \lambda \geq 1
$$

This shows that $\left(E_{V},(\cdot, \cdot)_{\lambda V}\right)$ is a Hilbert space for $\lambda \geq 1$. Furthermore, by virtue of the Sobolev embedding theorem, we have

$$
\|u\|_{s} \leq \gamma_{s}\|u\|_{H^{1}\left(\mathbb{R}^{N}\right)} \leq \gamma_{s} \gamma_{0}\|u\|_{\lambda V}, \quad \forall u \in E_{V}, \lambda \geq 1,2 \leq s \leq 2^{*}
$$

here and in the sequel, by $\|\cdot\|_{s}$ we denote the usual norm in space $L^{s}\left(\mathbb{R}^{N}\right)$.

Set $E=E_{V} \times E_{V}$, then $E$ is a Hilbert space with the inner product

$$
\left(z_{1}, z_{2}\right)_{\lambda \dagger}=\left(u_{1}, u_{2}\right)_{\lambda V}+\left(v_{1}, v_{2}\right)_{\lambda V}, \quad \forall z_{i}=\left(u_{i}, v_{i}\right) \in E, i=1,2,
$$

the corresponding norm is denoted by $\|\cdot\|_{\lambda_{\dagger}}$. Then we have

$$
\|z\|_{\lambda \dagger}^{2}=\|u\|_{\lambda V}^{2}+\|v\|_{\lambda V}^{2}, \quad \forall z=(u, v) \in E
$$

and

$$
\begin{aligned}
\|z\|_{s}^{s} & =\int_{\mathbb{R}^{N}}\left(u^{2}+v^{2}\right)^{s / 2} \mathrm{~d} x \leq 2^{(s-2) / 2}\left(\|u\|_{s}^{s}+\|v\|_{s}^{s}\right) \\
& \leq 2^{(s-2) / 2}\left(\gamma_{s} \gamma_{0}\right)^{s}\left(\|u\|_{\lambda V}^{s}+\|v\|_{\lambda V}^{s}\right)
\end{aligned}
$$




$$
\begin{aligned}
& \leq 2^{(s-2) / 2}\left(\gamma_{s} \gamma_{0}\right)^{s}\left(\|u\|_{\lambda V}^{2}+\|v\|_{\lambda V}^{2}\right)^{s / 2} \\
& =2^{(s-2) / 2}\left(\gamma_{s} \gamma_{0}\right)^{s}\|z\|_{\lambda \dagger}^{s}, \quad \forall s \in\left(2,2^{*}\right], z=(u, v) \in E .
\end{aligned}
$$

Let

$$
E^{-}=\left\{(u,-u): u \in E_{V}\right\}, \quad E^{+}=\left\{(u, u): u \in E_{V}\right\} .
$$

For any $z=(u, v) \in E$, set

$$
z^{-}=\left(\frac{u-v}{2}, \frac{v-u}{2}\right), \quad z^{+}=\left(\frac{u+v}{2}, \frac{u+v}{2}\right) .
$$

It is obvious that $z=z^{-}+z^{+}, z^{-}$and $z^{+}$are orthogonal with respect to the inner products $(\cdot, \cdot)_{L^{2}}$ and $(\cdot, \cdot)_{\lambda \dagger}$. Thus we have $E=E^{-} \oplus E^{+}$. By a simple calculation, one gets

$$
\frac{1}{2}\left(\left\|z^{+}\right\|_{\lambda \dagger}^{2}-\left\|z^{-}\right\|_{\lambda \dagger}^{2}\right)=\int_{\mathbb{R}^{N}}[\nabla u \cdot \nabla v+\lambda V(x) u v] \mathrm{d} x
$$

Therefore, the functional $\Phi_{\lambda}$ defined in (1.9) can be rewritten in a standard way

$$
\Phi_{\lambda}(z)=\frac{1}{2}\left(\left\|z^{+}\right\|_{\lambda \dagger}^{2}-\left\|z^{-}\right\|_{\lambda \dagger}^{2}\right)-\lambda \int_{\mathbb{R}^{N}} W(x, u, v) \mathrm{d} x, \quad \forall z=(u, v) \in E .
$$

Moreover,

$$
\begin{aligned}
\left\langle\Phi_{\lambda}^{\prime}(z), z\right\rangle= & \left\|z^{+}\right\|_{\lambda \dagger}^{2}-\left\|z^{-}\right\|_{\lambda \dagger}^{2} \\
& -\lambda \int_{\mathbb{R}^{N}}\left[W_{u}(x, u, v) u+W_{v}(x, u, v) v\right] \mathrm{d} x, \quad \forall z=(u, v) \in E .
\end{aligned}
$$

The following generalized linking theorem provides a convenient approach to get a $(C)_{c}$ sequence.

Let $X$ be a Hilbert space with $X=X^{-} \oplus X^{+}$and $X^{-} \perp X^{+}$. For a functional $\varphi \in$ $C^{1}(X, \mathbb{R}), \varphi$ is said to be weakly sequentially lower semi-continuous if for any $u_{n} \rightarrow u$ in $X$ one has $\varphi(u) \leq \liminf _{n \rightarrow \infty} \varphi\left(u_{n}\right)$, and $\varphi^{\prime}$ is said to be weakly sequentially continuous if $\lim _{n \rightarrow \infty}\left\langle\varphi^{\prime}\left(u_{n}\right), v\right\rangle=\left\langle\varphi^{\prime}(u), v\right\rangle$ for each $v \in X$.

Lemma 2.1 ([37, Theorem 4.5], [13, Theorem 2.1]) Let $X$ be a Hilbert space with $X=$ $X^{-} \oplus X^{+}$and $X^{-} \perp X^{+}$, and let $\varphi \in C^{1}(X, \mathbb{R})$ be of the form

$$
\varphi(u)=\frac{1}{2}\left(\left\|u^{+}\right\|^{2}-\left\|u^{-}\right\|^{2}\right)-\psi(u), \quad u=u^{+}+u^{-} \in X^{+} \perp X^{-} .
$$

Suppose that the following assumptions are satisfied:

(I1) $\psi \in C^{1}(X, \mathbb{R})$ is bounded from below and weakly sequentially lower semi-continuous;

(I2) $\psi^{\prime}$ is weakly sequentially continuous;

(I3) there exist $r>\rho>0$ and $e \in X^{+}$with $\|e\|=1$ such that

$$
\kappa:=\inf \varphi\left(S_{\rho}\right)>\sup \varphi(\partial Q)
$$


where

$$
S_{\rho}=\left\{u \in X^{+}:\|u\|=\rho\right\}, \quad Q=\left\{s e+v: v \in X^{-}, s \geq 0,\|s e+v\| \leq r\right\} .
$$

Then for some $c \geq \kappa$, there exists a sequence $\left\{u_{n}\right\} \subset X$ satisfying

$$
\varphi\left(u_{n}\right) \rightarrow c, \quad\left\|\varphi^{\prime}\left(u_{n}\right)\right\|\left(1+\left\|u_{n}\right\|\right) \rightarrow 0 .
$$

Such a sequence is called a Cerami sequence on the level c, or a $(C)_{c}$ sequence.

\section{Proofs of the theorems}

In this section, we give the proofs of Theorems 1.1 and 1.2.

From now on we assume without loss of generality that $x_{0}=0$ (see [47]), that is, $V(0)=0$, then $\lambda_{0}>1$ defined by (1.8) satisfies

$$
\sup _{\lambda^{1 / 2}|x| \leq 2 h_{0}}|V(x)| \leq h_{0}^{-2}, \quad \forall \lambda \geq \lambda_{0}
$$

Let

$$
\vartheta(x):= \begin{cases}\frac{1}{h_{0}}, & |x| \leq h_{0}, \\ \frac{h_{0}^{N-1}}{1-2^{-N}}\left[|x|^{-N}-\left(2 h_{0}\right)^{-N}\right], & h_{0}<|x| \leq 2 h_{0}, \\ 0, & |x|>2 h_{0} .\end{cases}
$$

Then $\vartheta \in H^{1}\left(\mathbb{R}^{N}\right)$, moreover,

$$
\begin{aligned}
& \|\nabla \vartheta\|_{2}^{2}=\int_{\mathbb{R}^{N}}|\nabla \vartheta(x)|^{2} \mathrm{~d} x \leq \frac{N^{2} \omega_{N}}{(N+2)\left(1-2^{-N}\right)^{2}} h_{0}^{N-4}, \\
& \|\vartheta\|_{2}^{2}=\int_{\mathbb{R}^{N}}|\vartheta(x)|^{2} \mathrm{~d} x \leq \frac{2 \omega_{N}}{\left(1-2^{-N}\right)^{2} N} h_{0}^{N-2}
\end{aligned}
$$

and

$$
\|\vartheta\|_{p}^{p}=\int_{\mathbb{R}^{N}}|\vartheta(x)|^{p} \mathrm{~d} x \geq \frac{\omega_{N}}{N} h_{0}^{N-p} .
$$

Let $e_{\lambda}(x)=\left(\vartheta\left(\lambda^{1 / 2} x\right), 0\right)$. Then we can prove the following lemma which is very important and crucial.

Lemma 3.1 Suppose that (V0), (V1), (W1), (W2), and (W3) are satisfied. Then

$$
\sup \left\{\Phi_{\lambda}\left(\zeta+s e_{\lambda}\right): \zeta=(w,-w) \in E^{-}, s \geq 0\right\} \leq \frac{b^{(2 \kappa-N) / 2}}{3^{\kappa} \sqrt{2} a_{1}\left(\gamma_{2^{*}} \gamma_{0}\right)^{N}} \lambda^{1-N / 2}, \quad \forall \lambda \geq \lambda_{0}
$$

Proof Note that $e_{\lambda}^{+}=\left(\vartheta\left(\lambda^{1 / 2} x\right) / 2, \vartheta\left(\lambda^{1 / 2} x\right) / 2\right)$, we have

$$
\begin{aligned}
\left\|e_{\lambda}^{+}\right\|_{\lambda \dagger}^{2} & =\frac{1}{2} \int_{\mathbb{R}^{N}}\left(\left|\nabla \vartheta\left(\lambda^{1 / 2} x\right)\right|^{2}+\lambda V(x)\left|\vartheta\left(\lambda^{1 / 2} x\right)\right|^{2}\right) \mathrm{d} x \\
& =\frac{1}{2} \lambda^{1-N / 2} \int_{\mathbb{R}^{N}}\left(|\nabla \vartheta|^{2}+V\left(\lambda^{-1 / 2} x\right)|\vartheta|^{2}\right) \mathrm{d} x
\end{aligned}
$$




$$
\begin{aligned}
& \leq \frac{1}{2} \lambda^{1-N / 2}\left(\|\nabla \vartheta\|_{2}^{2}+\|\vartheta\|_{2}^{2} \sup _{\lambda^{1 / 2}|x| \leq 2 h_{0}}|V(x)|\right) \\
& \leq \frac{1}{2} \lambda^{1-N / 2}\left(\|\nabla \vartheta\|_{2}^{2}+h_{0}^{-2}\|\vartheta\|_{2}^{2}\right), \quad \forall \lambda \geq \lambda_{0} .
\end{aligned}
$$

It follows from (W3), (1.7), (1.9), (3.3), (3.4), (3.5), and (3.7) that

$$
\begin{aligned}
\Phi_{\lambda}\left(\zeta+s e_{\lambda}\right) & =\frac{1}{2}\left(s^{2}\left\|e_{\lambda}^{+}\right\|_{\lambda^{\dagger}}^{2}-\left\|\zeta+s e_{\lambda}^{-}\right\|_{\lambda \dagger}^{2}\right)-\lambda \int_{\mathbb{R}^{N}} W\left(x, w+s \vartheta\left(\lambda^{1 / 2} x\right),-w\right) \mathrm{d} x \\
& \leq \frac{s^{2}}{2}\left\|e_{\lambda}^{+}\right\|_{\lambda^{\dagger}}^{2}-a_{0} \lambda s^{p} \int_{\mathbb{R}^{N}}\left|\vartheta\left(\lambda^{1 / 2} x\right)\right|^{p} \mathrm{~d} x \\
& \leq \lambda^{1-N / 2}\left\{\frac{s^{2}}{4}\left(\|\nabla \vartheta\|_{2}^{2}+h_{0}^{-2}\|\vartheta\|_{2}^{2}\right)-a_{0} s^{p}\|\vartheta\|_{p}^{p}\right\} \\
& \leq \lambda^{1-N / 2}\left[\frac{s^{2}}{4}\left(\frac{N^{3}+2(N+2)}{N(N+2)\left(1-2^{-N}\right)^{2}}\right) \omega_{N} h_{0}^{N-4}-\frac{a_{0} \omega_{N}}{N} s^{p} h_{0}^{N-p}\right] \\
& \leq \frac{(p-2) \omega_{N}}{4 N a_{0}^{2 /(p-2)}}\left[\frac{N^{3}+2(N+2)}{2(N+2) p\left(1-2^{-N}\right)^{2}}\right]^{p /(p-2)} h_{0}^{[(p-2) N-2 p] /(p-2)} \lambda^{1-N / 2} \\
& \leq \frac{b^{(2 \kappa-N) / 2}}{3^{\kappa} \sqrt{2} a_{1}\left(\gamma_{2^{*}} \gamma_{0}\right)^{N}} \lambda^{1-N / 2}, \quad \forall s \geq 0, \lambda \geq \lambda_{0}, \zeta=(w,-w) \in E^{-} .
\end{aligned}
$$

Now the conclusion of Lemma 3.1 follows by (3.8).

Applying Lemma 2.1, by standard arguments (see, e.g., [45]), we can prove the following lemma.

Lemma 3.2 Suppose that (V0), (V1), (W1), (W2), and (W3) are satisfied. Then there exist a constant $c_{\lambda} \in\left(0, \sup \left\{\Phi_{\lambda}\left(\zeta+s e_{\lambda}\right): \zeta=(w,-w) \in E^{-}, s \geq 0\right\}\right]$ and a sequence $\left\{z_{n}\right\}=$ $\left\{\left(u_{n}, v_{n}\right)\right\} \subset$ E satisfying

$$
\Phi_{\lambda}\left(z_{n}\right) \rightarrow c_{\lambda}, \quad\left\|\Phi_{\lambda}^{\prime}\left(z_{n}\right)\right\|\left(1+\left\|z_{n}\right\|\right) \rightarrow 0
$$

Lemma 3.3 Suppose that (V0), (V1), (W1), (W2), (W3), and (W4) are satisfied. Then any sequence $\left\{z_{n}\right\}=\left\{\left(u_{n}, v_{n}\right)\right\} \subset$ E satisfying (3.9) is bounded in $E$.

Proof By virtue of (W3), (2.6), and (3.9), one gets

$$
\begin{aligned}
2 c_{\lambda}+o(1) & =\left\|z_{n}^{+}\right\|_{\lambda \dagger}^{2}-\left\|z_{n}^{-}\right\|_{\lambda \dagger}^{2}-2 \lambda \int_{\mathbb{R}^{N}} W\left(x, u_{n}, v_{n}\right) \mathrm{d} x \\
& \leq\left\|z_{n}^{+}\right\|_{\lambda \dagger}^{2}-\left\|z_{n}^{-}\right\|_{\lambda \dagger}^{2}-2 a_{0} \lambda \int_{\mathbb{R}^{N}}\left|u_{n}+v_{n}\right|^{p} \mathrm{~d} x .
\end{aligned}
$$

To prove the boundedness of $\left\{z_{n}\right\}$, arguing by contradiction, suppose that $\left\|z_{n}\right\|_{\lambda \dagger} \rightarrow \infty$. Let $\xi_{n}=z_{n} /\left\|z_{n}\right\|_{\lambda \dagger}=\left(\varphi_{n}, \psi_{n}\right)$, then $\left\|\xi_{n}\right\|_{\lambda \dagger}=1$. If

$$
\delta:=\limsup _{n \rightarrow \infty} \sup _{y \in \mathbb{R}^{N}} \int_{B(y, 1)}\left|\xi_{n}^{+}\right|^{2} \mathrm{~d} x=0
$$


then by Lions' concentration compactness principle [48] or [49, Lemma 1.21], $\varphi_{n}+\psi_{n} \rightarrow 0$ in $L^{s}\left(\mathbb{R}^{N}\right)$ for $2<s<2^{*}$. Hence, it follows from (W4), (2.4), (3.10), and the Hölder inequality that

$$
\begin{aligned}
& \frac{\lambda}{2} \int_{\left|z_{n}\right| \leq R_{0}}\left|W_{u}\left(x, u_{n}, v_{n}\right)+W_{v}\left(x, u_{n}, v_{n}\right)\right|\left|u_{n}+v_{n}\right| \mathrm{d} x \\
& \leq \leq \frac{\lambda b}{6} \int_{\left|z_{n}\right| \leq R_{0}}\left|z_{n}\right|\left|u_{n}+v_{n}\right| \mathrm{d} x \\
& \leq \frac{\lambda b}{6} \int_{\mathbb{R}^{N} \backslash \mathcal{V}_{b}}\left|z_{n}\right|\left|u_{n}+v_{n}\right| \mathrm{d} x+\frac{\lambda b}{6} \int_{\mathcal{V}_{b}}\left|z_{n}\right|\left|u_{n}+v_{n}\right| \mathrm{d} x \\
& \leq \frac{\lambda b}{6}\left(\int_{\mathbb{R}^{N} \backslash \mathcal{V}_{b}}\left|z_{n}\right|^{2} \mathrm{~d} x\right)^{1 / 2}\left(\int_{\mathbb{R}^{N} \backslash \mathcal{V}_{b}}\left|u_{n}+v_{n}\right|^{2} \mathrm{~d} x\right)^{1 / 2} \\
& \quad+\frac{\lambda b\left[\operatorname{meas}\left(\mathcal{V}_{b}\right)\right]^{1 /(N+1)}}{6}\left(\int_{\mathcal{V}_{b}}\left|z_{n}\right|^{2(N+1) / N} \mathrm{~d} x\right)^{N / 2(N+1)} \\
& \quad \times\left(\int_{\mathcal{V}_{b}}\left|u_{n}+v_{n}\right|^{2(N+1) / N} \mathrm{~d} x\right)^{N / 2(N+1)} \\
& \leq \frac{1}{6}\left\|z_{n}\right\|_{\lambda \dagger}\left\|u_{n}+v_{n}\right\|_{\lambda V}+\frac{\lambda b\left[\operatorname{meas}\left(\mathcal{V}_{b}\right)\right]^{1 /(N+1)}}{6}\left\|z_{n}\right\|_{2(N+1) / N}\left\|u_{n}+v_{n}\right\|_{2(N+1) / N} \\
&= \frac{1}{6}\left\|z_{n}\right\|_{\lambda \dagger}\left\|u_{n}+v_{n}\right\|_{\lambda V}+\frac{\lambda b\left[\operatorname{meas}\left(\mathcal{V}_{b}\right)\right]^{1 /(N+1)}}{6}\left\|\xi_{n}\right\|_{2(N+1) / N} \\
& \times\left\|\varphi_{n}+\psi_{n}\right\|_{2(N+1) / N}\left\|z_{n}\right\|_{\lambda \dagger}^{2} \\
& \leq {\left[\frac{1}{3}+o(1)\right]\left\|z_{n}\right\|_{\lambda \dagger}^{2} \cdot }
\end{aligned}
$$

From (2.6), (2.7), and (3.9), one has

$$
c_{\lambda}+o(1)=\lambda \int_{\mathbb{R}^{N}} \widetilde{W}\left(x, u_{n}, v_{n}\right) \mathrm{d} x
$$

Let $\kappa^{\prime}=\kappa /(\kappa-1)$, then $2<2 \kappa^{\prime}<2^{*}$. Hence, by virtue of (W4), (3.12), and the Hölder inequality, one gets

$$
\begin{aligned}
\frac{\lambda}{2} \int_{\left|z_{n}\right| \geq R_{0}} \frac{\left|W_{u}\left(x, u_{n}, v_{n}\right)+W_{v}\left(x, u_{n}, v_{n}\right)\right|\left|u_{n}+v_{n}\right|}{\left\|z_{n}\right\|_{\lambda \dagger}^{2}} \mathrm{~d} x \\
=\frac{\lambda}{2} \int_{\left|z_{n}\right| \geq R_{0}} \frac{\left|W_{u}\left(x, u_{n}, v_{n}\right)+W_{v}\left(x, u_{n}, v_{n}\right)\right|\left|\xi_{n}\right|\left|\varphi_{n}+\psi_{n}\right|}{\left|z_{n}\right|} \mathrm{d} x \\
\leq \frac{\lambda}{2}\left(\int_{\left|z_{n}\right| \geq R_{0}}\left|\frac{W_{u}\left(x, u_{n}, v_{n}\right)+W_{v}\left(x, u_{n}, v_{n}\right)}{z_{n}}\right|^{\kappa} \mathrm{d} x\right)^{1 / \kappa}\left(\int_{\left|z_{n}\right| \geq R_{0}}\left|\xi_{n}\right|^{2 \kappa^{\prime}} \mathrm{d} x\right)^{1 / 2 \kappa^{\prime}} \\
\quad \times\left(\int_{\left|z_{n}\right| \geq R_{0}}\left|\varphi_{n}+\psi_{n}\right|^{2 \kappa^{\prime}} \mathrm{d} x\right)^{1 / 2 \kappa^{\prime}} \\
\leq \frac{\lambda}{2}\left(a_{1} \int_{\left|z_{n}\right| \geq R_{0}} \widetilde{W}\left(x, u_{n}, v_{n}\right) \mathrm{d} x\right)^{1 / \kappa}\left\|\xi_{n}\right\|_{2 \kappa^{\prime}}\left\|\varphi_{n}+\psi_{n}\right\|_{2 \kappa^{\prime}} \\
\leq \lambda^{(\kappa-1) / \kappa}\left(c_{\lambda} a_{1}\right)^{1 / \kappa}\left\|\xi_{n}\right\|_{2 \kappa^{\prime}}\left\|\varphi_{n}+\psi_{n}\right\|_{2 \kappa^{\prime}}=o(1) .
\end{aligned}
$$


Combining (3.11) with (3.13) and using (1.10), (3.9), and (3.10), we have

$$
\begin{aligned}
\frac{1}{2}+o(1) \leq & \frac{\left\|z_{n}^{+}\right\|_{\lambda \dagger}^{2}-\left\langle\Phi_{\lambda}^{\prime}\left(z_{n}\right), z_{n}^{+}\right\rangle}{\left\|z_{n}\right\|_{\lambda \dagger}^{2}} \\
= & \frac{\lambda}{2} \int_{\mathbb{R}^{N}} \frac{\left[W_{u}\left(x, u_{n}, v_{n}\right)+W_{v}\left(x, u_{n}, v_{n}\right)\right]\left(u_{n}+v_{n}\right)}{\left\|z_{n}\right\|_{\lambda \dagger}^{2}} \mathrm{~d} x \\
= & \frac{\lambda}{2} \int_{\left|z_{n}\right| \leq R_{0}} \frac{\left[W_{u}\left(x, u_{n}, v_{n}\right)+W_{v}\left(x, u_{n}, v_{n}\right)\right]\left(u_{n}+v_{n}\right)}{\left\|z_{n}\right\|_{\lambda^{\dagger}}^{2}} \mathrm{~d} x \\
& +\frac{\lambda}{2} \int_{\left|z_{n}\right|>R_{0}} \frac{\left[W_{u}\left(x, u_{n}, v_{n}\right)+W_{v}\left(x, u_{n}, v_{n}\right)\right]\left(u_{n}+v_{n}\right)}{\left\|z_{n}\right\|_{\lambda \dagger}^{2}} \mathrm{~d} x \\
\leq & \frac{1}{3}+o(1) .
\end{aligned}
$$

This contradiction shows that $\delta>0$.

Going if necessary to a subsequence, we may assume the existence of $k_{n} \in \mathbb{Z}^{N}$ such that $\int_{B_{1+\sqrt{N}}\left(k_{n}\right)}\left|\xi_{n}^{+}\right|^{2} \mathrm{~d} x>\frac{\delta}{2}$. Let $\zeta_{n}(x)=\xi_{n}\left(x+k_{n}\right)$. Then

$$
\int_{B_{1+\sqrt{N}}(0)}\left|\zeta_{n}^{+}\right|^{2} \mathrm{~d} x>\frac{\delta}{2}
$$

Now we define $\tilde{z}_{n}(x)=\left(\tilde{u}_{n}, \tilde{v}_{n}\right)=z_{n}\left(x+k_{n}\right)$, then $\tilde{z}_{n} /\left\|z_{n}\right\|_{\lambda \dagger}=\zeta_{n}$ and $\left\|\zeta_{n}\right\|_{H^{1}\left(\mathbb{R}^{N}\right)}^{2}=\left\|\xi_{n}\right\|_{H^{1}\left(\mathbb{R}^{N}\right)}^{2}$. Passing to a subsequence, we have $\zeta_{n} \rightarrow \zeta$ in $E, \zeta_{n} \rightarrow \zeta$ in $L_{\text {loc }}^{s}\left(\mathbb{R}^{N}\right), 2 \leq s<2^{*}$, and $\zeta_{n} \rightarrow \zeta$ a.e. on $\mathbb{R}^{N}$. Obviously, (3.15) implies that $\zeta^{+} \neq 0$. For a.e. $x \in\left\{y \in \mathbb{R}^{N}: \zeta^{+}(y) \neq 0\right\}:=\Omega$, we have $\lim _{n \rightarrow \infty}\left|\tilde{u}_{n}(x)+\tilde{v}_{n}(x)\right|=\infty$. Hence, it follows from (2.6), (3.9), (W3), and Fatou's lemma that

$$
\begin{aligned}
0 & =\lim _{n \rightarrow \infty} \frac{c+o(1)}{\left\|z_{n}\right\|_{\lambda^{\dagger}}^{2}}=\lim _{n \rightarrow \infty} \frac{\Phi_{\lambda}\left(z_{n}\right)}{\left\|z_{n}\right\|_{\lambda^{\dagger}}^{2}} \\
& =\lim _{n \rightarrow \infty}\left[\frac{1}{2}\left(\left\|\xi_{n}^{+}\right\|_{\lambda \dagger}^{2}-\left\|\xi_{n}^{-}\right\|_{\lambda^{\dagger}}^{2}\right)-\lambda \int_{\mathbb{R}^{N}} \frac{W\left(x, u_{n}, v_{n}\right)}{\left\|z_{n}\right\|_{\lambda^{\dagger}}^{2}} \mathrm{~d} x\right] \\
& =\lim _{n \rightarrow \infty}\left[\frac{1}{2}\left(\left\|\xi_{n}^{+}\right\|_{\lambda^{\dagger}}^{2}-\left\|\xi_{n}^{-}\right\|_{\lambda_{\dagger}}^{2}\right)-\lambda \int_{\mathbb{R}^{N}} \frac{W\left(x+k_{n}, \tilde{u}_{n}, \tilde{v}_{n}\right)}{\left|\tilde{z}_{n}^{+}\right|^{2}}\left|\zeta_{n}^{+}\right|^{2} \mathrm{~d} x\right] \\
& =\lim _{n \rightarrow \infty}\left[\frac{1}{2}\left(\left\|\xi_{n}^{+}\right\|_{\lambda \dagger}^{2}-\left\|\xi_{n}^{-}\right\|_{\lambda_{\dagger}}^{2}\right)-2 \lambda \int_{\mathbb{R}^{N}} \frac{W\left(x+k_{n}, \tilde{u}_{n}, \tilde{v}_{n}\right)}{\left|\tilde{u}_{n}+\tilde{v}_{n}\right|^{2}}\left|\zeta_{n}^{+}\right|^{2} \mathrm{~d} x\right] \\
& \leq \frac{1}{2}-2 \lambda \int_{\Omega} \liminf _{n \rightarrow \infty} \frac{W\left(x+k_{n}, \tilde{u}_{n}, \tilde{v}_{n}\right)}{\left|\tilde{u}_{n}+\tilde{v}_{n}\right|^{2}}\left|\zeta_{n}^{+}\right|^{2} \mathrm{~d} x=-\infty .
\end{aligned}
$$

This contradiction shows that $\left\|z_{n}\right\|_{\lambda \uparrow}$ is bounded.

Proof of Theorem 1.2 Applying Lemmas 3.1, 3.2, and 3.3, we deduce that there exists a bounded sequence $\left\{z_{n}\right\}=\left\{\left(u_{n}, v_{n}\right)\right\} \subset E$ satisfying (3.9) and (3.10) with

$$
c_{\lambda} \leq \frac{b^{(2 \kappa-N) / 2}}{3^{\kappa} \sqrt{2} a_{1}\left(\gamma_{2} * \gamma_{0}\right)^{N}} \lambda^{1-N / 2}, \quad \forall \lambda \geq \lambda_{0} .
$$

Going if necessary to a subsequence, we can assume that $z_{n} \rightarrow z_{\lambda}=\left(u_{\lambda}, v_{\lambda}\right)$ in $\left(E,\|\cdot\|_{\lambda_{\dagger}}\right)$, and $\Phi_{\lambda}^{\prime}\left(z_{n}\right) \rightarrow 0$. Next, we prove that $z_{\lambda} \neq 0$. 
Arguing by contradiction, suppose that $z_{\lambda}=0$, i.e. $z_{n} \rightarrow 0$ in $E$, and so $u_{n} \rightarrow 0, v_{n} \rightarrow 0$ in $L_{\text {loc }}^{s}\left(\mathbb{R}^{N}\right), 2 \leq s<2^{*}$, and $u_{n} \rightarrow 0, v_{n} \rightarrow 0$ a.e. on $\mathbb{R}^{N}$. Since $\mathcal{V}_{b}$ is a set of finite measure, we have

$$
\left\|u_{n}+v_{n}\right\|_{2}^{2}=\int_{\mathbb{R}^{N} \backslash \mathcal{V}_{b}}\left|u_{n}+v_{n}\right|^{2} \mathrm{~d} x+\int_{\mathcal{V}_{b}}\left|u_{n}+v_{n}\right|^{2} \mathrm{~d} x \leq \frac{1}{\lambda b}\left\|u_{n}+v_{n}\right\|_{\lambda V}^{2}+o(1)
$$

and

$$
\left\|z_{n}\right\|_{2}^{2}=\int_{\mathbb{R}^{N} \backslash \mathcal{V}_{b}}\left(u_{n}^{2}+v_{n}^{2}\right) \mathrm{d} x+\int_{\mathcal{V}_{b}}\left(u_{n}^{2}+v_{n}^{2}\right) \mathrm{d} x \leq \frac{1}{\lambda b}\left\|z_{n}\right\|_{\lambda \dagger}^{2}+o(1) .
$$

For $s \in\left(2,2^{*}\right)$, it follows from (2.3), (2.5), (3.17), (3.18), and the Hölder inequality that

$$
\begin{aligned}
\left\|u_{n}+v_{n}\right\|_{s}^{s} & \leq\left\|u_{n}+v_{n}\right\|_{2}^{2\left(2^{*}-s\right) /\left(2^{*}-2\right)}\left\|u_{n}+v_{n}\right\|_{2^{*}}^{2^{*}(s-2) /\left(2^{*}-2\right)} \\
& \leq\left(\gamma_{2^{*}} \gamma_{0}\right)^{2^{*}(s-2) /\left(2^{*}-2\right)}(\lambda b)^{-\left(2^{*}-s\right) /\left(2^{*}-2\right)}\left\|u_{n}+v_{n}\right\|_{\lambda V}^{s}+o(1)
\end{aligned}
$$

and

$$
\begin{aligned}
\left\|z_{n}\right\|_{s}^{s} & \leq\left\|z_{n}\right\|_{2}^{2\left(2^{*}-s\right) /\left(2^{*}-2\right)}\left\|z_{n}\right\|_{2^{*}}^{2^{*}(s-2) /\left(2^{*}-2\right)} \\
& \leq 2^{(s-2) / 2}\left(\gamma_{2^{*}} \gamma_{0}\right)^{2^{*}(s-2) /\left(2^{*}-2\right)}(\lambda b)^{-\left(2^{*}-s\right) /\left(2^{*}-2\right)}\left\|z_{n}\right\|_{\lambda \dagger}^{s}+o(1) .
\end{aligned}
$$

According to (W4), (3.10), (3.17), and (3.18), one gets

$$
\begin{aligned}
& \frac{\lambda}{2} \int_{\left|z_{n}\right| \leq R_{0}}\left|W_{u}\left(x, u_{n}, v_{n}\right)+W_{v}\left(x, u_{n}, v_{n}\right)\right|\left|u_{n}+v_{n}\right| \mathrm{d} x \\
& \quad \leq \frac{\lambda b}{6} \int_{\left|z_{n}\right| \leq R_{0}}\left|z_{n}\right|\left|u_{n}+v_{n}\right| \mathrm{d} x \\
& \quad \leq \frac{\lambda b}{6}\left\|z_{n}\right\|_{2}\left\|u_{n}+v_{n}\right\|_{2} \leq \frac{1}{6}\left\|z_{n}\right\|_{\lambda \dagger}\left\|u_{n}+v_{n}\right\|_{\lambda V} \\
& \quad \leq \frac{1}{3}\left\|z_{n}^{+}\right\|_{\lambda \dagger}^{2}+o(1) .
\end{aligned}
$$

By virtue of (1.9), (1.10), and (3.9), we have

$$
\Phi_{\lambda}\left(u_{n}\right)-\frac{1}{2}\left\langle\Phi_{\lambda}^{\prime}\left(u_{n}\right), u_{n}\right\rangle=\lambda \int_{\mathbb{R}^{N}} \widetilde{W}\left(x, u_{n}, v_{n}\right) \mathrm{d} x=c_{\lambda}+o(1) .
$$

Using (W4), (3.16), (3.19), (3.20) with $s=2 \kappa /(\kappa-1)$, and (3.22), we obtain

$$
\begin{aligned}
& \frac{\lambda}{2} \int_{\left|u_{n}\right|>R_{0}}\left|W_{u}\left(x, u_{n}, v_{n}\right)+W_{v}\left(x, u_{n}, v_{n}\right)\right|\left|u_{n}+v_{n}\right| \mathrm{d} x \\
& \leq \frac{\lambda}{2}\left(\int_{\left|u_{n}\right|>R_{0}}\left(\frac{\left|W_{u}\left(x, u_{n}, v_{n}\right)+W_{v}\left(x, u_{n}, v_{n}\right)\right|}{\left|z_{n}\right|}\right)^{\kappa} \mathrm{d} x\right)^{1 / \kappa}\left\|z_{n}\right\|_{s}\left\|u_{n}+v_{n}\right\|_{s} \\
& \leq \frac{2^{(s-2) / 2 s}}{2}\left(\gamma_{2^{*}} * \gamma_{0}\right)^{2 \cdot 2^{*}(s-2) / s\left(2^{*}-2\right)} \lambda\left(a_{1} \int_{\left|u_{n}\right|>R_{0}} \widetilde{W}\left(x, u_{n}, v_{n}\right) \mathrm{d} x\right)^{1 / \kappa} \\
& \quad \times(\lambda b)^{-2\left(2^{*}-s\right) / s\left(2^{*}-2\right)}\left\|z_{n}\right\|_{\lambda \dagger}\left\|u_{n}+v_{n}\right\|_{\lambda V}+o(1)
\end{aligned}
$$




$$
\begin{aligned}
& \leq \frac{1}{2}\left(\sqrt{2} a_{1}\right)^{1 / \kappa}\left(\gamma_{2^{*}} \gamma_{0}\right)^{N / \kappa} \lambda^{1-1 / \kappa} c_{\lambda}^{1 / \kappa}(\lambda b)^{(N-2 \kappa) / 2 \kappa}\left\|z_{n}\right\|_{\lambda \dagger}\left\|u_{n}+v_{n}\right\|_{\lambda V}+o(1) \\
& \leq \frac{\left(\sqrt{2} a_{1}\right)^{1 / \kappa}\left(\gamma_{2^{*}} \gamma_{0}\right)^{N / \kappa}}{b^{(2 \kappa-N) / 2 \kappa}}\left[\lambda^{(N-2) / 2} c_{\lambda}\right]^{1 / \kappa}\left\|z_{n}^{+}\right\|_{\lambda \dagger}^{2}+o(1) \\
& \leq \frac{1}{3}\left\|z_{n}^{+}\right\|_{\lambda \dagger}^{2}+o(1)
\end{aligned}
$$

which, together with (1.10), (3.9), (3.21), and (3.23), yields

$$
\begin{aligned}
o(1) & =\left\langle\Phi_{\lambda}^{\prime}\left(z_{n}\right), z_{n}^{+}\right\rangle=\left\|z_{n}^{+}\right\|_{\lambda \dagger}^{2}-\frac{\lambda}{2} \int_{\mathbb{R}^{N}}\left[W_{u}\left(x, u_{n}, v_{n}\right)+W_{v}\left(x, u_{n}, v_{n}\right)\right]\left(u_{n}+v_{n}\right) \mathrm{d} x \\
& \geq \frac{1}{3}\left\|z_{n}^{+}\right\|_{\lambda \dagger}^{2}+o(1),
\end{aligned}
$$

resulting in the fact that $\left\|z_{n}^{+}\right\|_{\lambda \dagger} \rightarrow 0$, which contradicts (3.10). Thus $z_{\lambda} \neq 0$. By a standard argument, we easily verify that $\Phi_{\lambda}^{\prime}\left(z_{\lambda}\right)=0$ and $\Phi_{\lambda}\left(z_{\lambda}\right) \leq c_{\lambda}$. Then $z_{\lambda}=\left(u_{\lambda}, v_{\lambda}\right)$ is a nontrivial solution of (2.1), moreover,

$$
c_{\lambda} \geq \Phi_{\lambda}\left(z_{\lambda}\right)=\Phi_{\lambda}\left(z_{\lambda}\right)-\frac{1}{2}\left\langle\Phi_{\lambda}^{\prime}\left(z_{\lambda}\right), z_{\lambda}\right\rangle=\lambda \int_{\mathbb{R}^{N}} \widetilde{W}\left(x, u_{\lambda}, v_{\lambda}\right) \mathrm{d} x
$$

\section{Theorem 1.1 is a direct consequence of Theorem 1.2.}

\section{Competing interests}

The authors declare that they have no competing interests.

\section{Authors' contributions}

All authors contributed equally to the writing of this paper. All authors read and approved the final manuscript.

\section{Author details}

'School of Mathematics and Statistics, Central South University, Changsha, Hunan 410083, P.R. China. ${ }^{2}$ Department of Mathematics, Xiangnan University, Chenzhou, Hunan 423000, P.R. China.

\section{Acknowledgments}

This work is partially supported by the NNSF (No. 11471278) of China, Scientific Research Fund of Hunan Provincial Education Department (12C0895), and the Construct Program of the Key Discipline in Hunan Province.

\section{Received: 1 June 2014 Accepted: 21 August 2014 Published online: 25 September 2014}

\section{References}

1. Clément, P, van der Vorst, RCAM: On a semilinear elliptic system. Differ. Integral Equ. 8, 1317-1329 (1995)

2. Clément, $\mathrm{P}$, de Figueiredo, DG, Mitidieri, E: Positive solutions of semilinear elliptic systems. Commun. Partial Differ. Equ. 17, 923-940 (1992)

3. de Figueiredo, DG, Felmer, PL: On superquadratic elliptic systems. Trans. Am. Math. Soc. 343, 97-116 (1994)

4. de Figueiredo, DG, Ding, YH: Strongly indefinite functionals and multiple solutions of elliptic systems. Trans. Am. Math. Soc. 355, 2973-2989 (2003)

5. Hulshof, J, van der Vorst, RCAM: Differential systems with strongly variational structure. J. Funct. Anal. 114, 32-58 (1993)

6. Kryszewski, W, Szulkin, A: An infinite dimensional Morse theory with applications. Trans. Am. Math. Soc. 349 3181-3234 (1997)

7. Bartsch, T, de Fiqueiredo, DG: Infinitely many solutions of nonlinear elliptic systems. In: Topics in Nonlinear Analysis. Progress in Nonlinear Differential Equations and Their Applications, vol. 35, pp. 51-67. Birkhäuser, Basel (1999)

8. de Figueiredo, DG, Yang, J: Decay, symmetry and existence of solutions of semilinear elliptic systems. Nonlinear Anal. 33, 211-234 (1998)

9. Li, G, Yang, J: Asymptotically linear elliptic systems. Commun. Partial Differ. Equ. 29, 925-954 (2004)

10. Ávila, Al, Yang, J: Multiple solutions of nonlinear elliptic systems. NoDEA Nonlinear Differ. Equ. Appl. 12, 459-479 (2005)

11. Avila, Al, Yang, J: On the existence and shape of least energy solutions for some elliptic systems. J. Differ. Equ. 191 348-376 (2003)

12. Bartsch, T, Ding, YH: Deformation theorems on non-metrizable vector spaces and applications to critical point theory. Math. Nachr. 279, 1267-1288 (2006) 
13. Li, GB, Szulkin, A: An asymptotically periodic Schrödinger equation with indefinite linear part. Commun. Contemp. Math. 4, 763-776 (2002)

14. Kryszewki, W, Szulkin, A: Generalized linking theorem with an application to semilinear Schrödinger equation. Adv. Differ. Equ. 3, 441-472 (1998)

15. Sirakov, B: On the existence of solutions of Hamiltonian elliptic systems in $\mathbb{R}^{N}$. Adv. Differ. Equ. 5, 1445-1464 (2000)

16. Zhang, J, Tang, XH, Zhang, W: Ground-state solutions for superquadratic Hamiltonian elliptic systems with gradient terms. Nonlinear Anal. 95, 1-10 (2014)

17. Zhang, J, Qin, WP, Zhao, FK: Existence and multiplicity of solutions for asymptotically linear nonperiodic Hamiltonian elliptic system. J. Math. Anal. Appl. 399, 433-441 (2013)

18. Zhao, F, Zhao, L, Ding, Y: Infinitely many solutions for asymptotically linear periodic Hamiltonian elliptic systems. ESAIM Control Optim. Calc. Var. 16, 77-91 (2010)

19. Zhao, F, Zhao, L, Ding, Y: Multiple solution for a superlinear and periodic elliptic system on $\mathbb{R}^{N}$. Z. Angew. Math. Phys. 62, 495-511 (2011)

20. Ambrosetti, A, Badiale, M, Cingolani, S: Semiclassical states of nonlinear Schrödinger equations. Arch. Ration. Mech. Anal. 140, 285-300 (1997)

21. Floer, A, Weinstein, A: Nonspreading wave pachets for the packets for the cubic Schrödinger with a bounded potential. J. Funct. Anal. 69, 397-408 (1986)

22. Oh, YG: Existence of semiclassical bound states of nonlinear Schrödinger equations with potentials of the class $(V)_{\alpha}$. Commun. Partial Differ. Equ. 13, 1499-1519 (1988)

23. Oh, YG: On positive multi-lump bound states of nonlinear Schrödinger equations under multiple well potential. Commun. Math. Phys. 131, 223-253 (1990)

24. del Pino, M, Felmer, P: Multipeak bound states of nonlinear Schrödinger equations. Ann. Inst. Henri Poincaré, Anal. Non Linéaire 15, 127-149 (1998)

25. del Pino, M, Felmer, P: Semi-classical states of nonlinear Schrödinger equations: a variational reduction method. Math. Ann. 324, 1-32 (2002)

26. Rabinowitz, PH: On a class of nonlinear Schrödinger equations. Z. Angew. Math. Phys. 43, $270-291$ (1992)

27. Zhang, J, Zhao, FK: Multiple solutions for a semiclassical Schrödinger equation. Nonlinear Anal. 75, 1834-1842 (2012)

28. Lin, $\mathrm{X}$, Tang, XH: Semiclassical solutions of perturbed $p$-Laplacian equations with critical nonlinearity. J. Math. Anal. Appl. 413, 439-449 (2014)

29. Ding, YH, Lee, C, Zhao, FK: Semiclassical limits of ground state solutions to Schrödinger systems. Calc. Var. (2013). doi:10.1007/s00526-013-0693-6

30. Ramos, M: On singular perturbations of superlinear elliptic systems. J. Math. Anal. Appl. 352, 246-258 (2009)

31. Ramos, M, Tavares, H: Solutions with multiple spike patterns for an elliptic system. Calc. Var. 31, 1-25 (2008)

32. Pistoia, A, Ramos, M: Locating the peaks of the least energy solutions to an elliptic system with Neumann boundary conditions. J. Differ. Equ. 201, 160-176 (2004)

33. Sirakov, B, Soares, SHM: Soliton solutions to systems of coupled Schrödinger equations of Hamiltonian type. Trans. Am. Math. Soc. 362, 5729-5744 (2010)

34. Xiao, L, Wang, J, Fan, M, Zhang, F: Existence and multiplicity of semiclassical solutions for asymptotically Hamiltonian elliptic systems. J. Math. Anal. Appl. 399, 340-351 (2013)

35. Zhang, J, Tang, XH, Zhang, W: Semiclassical solutions for a class of Schrödinger system with magnetic potentials. J. Math. Anal. Appl. 414, 357-371 (2014)

36. Ding, YH, Lee, C: Multiple solutions of Schrödinger equations with indefinite linear part and super or asymptotically linear terms. J. Differ. Equ. 222, 137-163 (2006)

37. Ding, YH: Variational Methods for Strongly Indefinite Problems. World Scientific, Hackensack (2008)

38. Ding, YH, Lee, C: Periodic solutions of an infinite dimensional Hamiltonian system. Rocky Mt. J. Math. 35, 1881-1908 (2005)

39. Qin, DD, Tang, XH, Jian, Z: Multiple solutions for semilinear elliptic equations with sign-changing potential and nonlinearity. Electron. J. Differ. Equ. 2013, 207 (2013)

40. Tang, XH: Infinitely many solutions for semilinear Schrödinger equation with sign-changing potential and nonlinearity. J. Math. Anal. Appl. 401, 407-415 (2013)

41. Tang, XH: New super-quadratic conditions on ground state solutions for superlinear Schrödinger equation. Adv. Nonlinear Stud. 14, 349-361 (2014)

42. Tang, XH: New conditions on nonlinearity for a periodic Schrödinger equation having zero as spectrum. J. Math. Anal. Appl. 413, 392-410 (2014)

43. Tang, XH: Non-Nehari manifold method for superlinear Schrödinger equation. Taiwan. J. Math. (2014). doi:10.11650/tjm.18.2014.3541

44. Zhang, RM, Chen, J, Zhao, FK: Multiple solutions for superlinear elliptic systems of Hamiltonian type. Discrete Contin. Dyn. Syst., Ser. A 30, 1249-1262 (2011)

45. Liao, FF, Tang, XH, Zhang, J: Existence of solutions for periodic elliptic system with general superlinear nonlinearity. Z. Angew. Math. Phys. (2014). doi:10.1007/s00033-014-0425-6

46. Sirakov, B: Standing wave solutions of the nonlinear Schrödinger equations in $\mathbb{R}^{N}$. Ann. Mat. 183, 73-83 (2002)

47. Ding, YH, Wei, JC: Semiclassical states for nonlinear Schrödinger equations with sign-changing potentials. J. Funct. Anal. 251, 546-572 (2007)

48. Lions, PL: The concentration-compactness principle in the calculus of variations. The locally compact case, part 2. Ann. Inst. Henri Poincaré, Anal. Non Linéaire 1, 223-283 (1984)

49. Willem, M: Minimax Theorems. Birkhäuser, Boston (1996)

doi:10.1186/s13661-014-0208-1

Cite this article as: Liao et al.: Semi-classical solutions of perturbed elliptic system with general superlinear nonlinearity. Boundary Value Problems 2014 2014:208. 